\title{
miR-21 in Human Cardiomyopathies
}

\author{
Surina ${ }^{1}$, Rosaria Anna Fontanella ${ }^{1}$, Lucia Scisciola ${ }^{1}$, Raffaele Marfella ${ }^{1,2}$, \\ Giuseppe Paolisso ${ }^{1,2}$ and Michelangela Barbieri ${ }^{1 *}$ \\ ${ }^{1}$ Department of Advanced Medical and Surgical Sciences, University of Campania "Luigi Vanvitelli", Naples, Italy, \\ ${ }^{2}$ Mediterrannea Cardiocentro, Napoli, Italy
}

miR-21 is a 22-nucleotide long microRNA that matches target mRNAs in a complementary base pairing fashion and regulates gene expression by repressing or degrading target mRNAs. miR-21 is involved in various cardiomyopathies, including heart failure, dilated cardiomyopathy, myocardial infarction, and diabetic cardiomyopathy. Expression levels of miR-21 notably change in both heart and circulation and provide cardiac protection after heart injury. In the meantime, miR-21 also tightly links to cardiac dysfunctions such as cardiac hypertrophy and fibrosis. This review focuses on the miR-21 expression pattern and its functions in diseased-heart and further discusses the feasibility of miR-21 as a biomarker and therapeutic target in cardiomyopathies.

Keywords: miR-21, cardiomyopathies, biomarkers, targeted therapy, fibrosis

\section{OPEN ACCESS}

Edited by:

Emilio Clementi,

University of Milan, Italy

Reviewed by:

Konrad Urbanek,

Magna Græcia University of

Catanzaro, Italy

Francesco Squadrito,

University of Messina, Italy

${ }^{*}$ Correspondence:

Michelangela Barbieri

michelangela.barbieri@unicampania.it

Specialty section:

This article was submitted to Cardiovascular Metabolism,

a section of the journal

Frontiers in Cardiovascular Medicine

Received: 30 August 2021

Accepted: 30 September 2021

Published: 27 October 2021

Citation:

Surina, Fontanella RA, Scisciola L,

Marfella R, Paolisso G and Barbieri M (2021) miR-21 in Human

Cardiomyopathies.

Front. Cardiovasc. Med. 8:767064.

doi: 10.3389/fcvm.2021.767064

\section{INTRODUCTION}

Cardiomyopathy refers to heart muscle diseases that show functional and structural myocardial abnormalities such as increasing thickness, dilation, and stiffness (1). As a result, the heart muscle is weakened, reducing the capacity to pump blood out, which often leads to irregular heartbeat and heart failure. Cardiomyopathy is complex and heterogeneous heart disease, causes immense health and economic burdens worldwide. Despite the progress already made, prevention and control of cardiomyopathies are still challenging. There is still lagging continuous innovation in pharmaceuticals, medical devices, and diagnostics. It is necessary to explore further insights into molecular and pathological processes of the diseased heart to develop early diagnostic and new therapeutic strategies.

Over the past two decades, numerous research studies in biomarker identification and targeted therapy development have brought microRNA(miRNAs) research to the fore. miRNAs are short non-coding RNAs that repress target mRNAs expression by altering transcript stability or impacting mRNA translation (2). microRNAs play essential roles in the molecular network of cardiac development and cardiac disease pathogenesis (3). Of them, the most studied miRNA is miR-21, which is suggested as a predictive and diagnostic maker for many cardiac disorders, including heart failure, myocardial infarction, coronary artery disease, diabetic cardiomyopathy, and myocardial ischemic reperfusion injury (4-8). Yet its expression trends are inconsistent in different studies and vary depending on expression sites and disease progression (9). Cardiac stresses commonly induce miR-21 expression, mediating stress-related signaling pathways to protect cardiomyocytes from apoptosis. However, miRNA-21 up-regulation also results in myocardial fibrosis by activating cardiac fibroblasts to myofibroblasts and causing further damage to the heart (10). In addition, it was reported that miR-21 was not essential for pathological cardiac remodeling because miR-21 knockout and inhibition in mice both displayed cardiac hypertrophy and fibrosis in response to various cardiac stresses (11). These contradictory findings may indicate the multiple roles of miR-21 in cardiomyopathies, and the pathological activities may depend on 
the cell types and disease conditions. In this review, we present an update of current findings of miR-21 and further discussion on the contradictory role of miR-21 in the pathogenesis of cardiac diseases and its future therapeutic perspectives (Figure 1).

\section{OVERVIEW OF miR-21}

microRNAs (miRNAs), a family of short ( $\sim 22$ nucleotides) noncoding RNAs that negatively regulate gene expression by binding 3' untranslated region (3'UTR) of their target mRNAs, leading to translational repression and degradation (12). To date, over 2,000 miRNAs have been identified in humans since the first miRNA, lin-4 was discovered in Caenorhabditis elegans in 1993 (13). Of them, miR-21 has been gained the most interest of researchers, and the studies include a wide range of diseases covering cancer, metabolic diseases, and viral pathogenesis (14).

\section{Biogenesis}

The human miR-21 gene has 3,433 nucleotides, located in chromosome 17q23.2, an intergenic region with the 3' UTR end of the Transmembrane Protein 49 (TMEM 49) (also known as Human Vacuole Membrane Protein 1, VMP-1) (15). miRNAs share a common biosynthetic pathway. The biogenic processing steps of miR-21 were first characterized in 2004 (16). miR-21 gene has two transcription sites, T1 (the minor transcription site) and T2 (the primary transcription site), and both are utilized for the initiation of transcription. RNA Pol II enzyme initiates miR-21 transcription and synthesizes full-length $(\sim 3.5$ $\mathrm{kb}$ ) primary miR-21 (pri-miR-21) in the nucleus (17). Following transcription, primary miR-21 requires two cleavage steps to become a mature and functional miRNA. First, pri-miR-21 is cleaved into $\approx 70$-nt-long intermediate precursor miR-21(premiR-21) by the microprocessor complex that contains RNase III
Drosha and a dsRNA binding protein DGCR8 $(18,19)$. Exportin 5 transports pre-miR-21 in a Ran-GTPase-dependent manner from nuclear to cytoplasm, where maturation can be completed (20). Upon export to the cytoplasm, a second RNase III enzyme, Dicer, removes the terminal loop from pre-miRNA and releases a small 22-nucleotide miRNA duplex. Following miRNA duplex loading, the precursor RNA-induced silencing complex (preRISC) quickly degrades the "passenger" strand to form a mature miRNA- RISC complex (21).

\section{Physiological Functions}

It is shown that most human protein-coding genes contain one or more miRNA-binding sites, so that miRNAs strictly regulate protein expression (22). In miRNA- RISC complex, miRNAs serve as target recognition purpose. "miRNA seed" is a domain at 2-7 nucleotides position of the 5' end of miRNAs. It recognizes the 3' untranslated region (UTR) of mRNAs, and miRNAs bind to UTR with complementary base pairing fashion. Once locked on an mRNA, the RISC silencing complex promotes downregulation of the protein by repressing or degrading the mRNA (23).

\section{Targets Genes}

miR-21 is proven to be involved in the pathogenesis of cardiovascular diseases through regulating downstream target genes. To date, several genes are identified as miR-21 targets in cardiomyopathy. The most recognized two target genes that involve in anti-apoptosis are programmed cell death 4 (PDCD4) (24) and phosphatase and tensin homolog deleted from chromosome 10 (PTEN) (25). In cardiac fibrosis, the primary three target genes are SMAD family member 7 (Smad7) (26) and sprouty1/2 (SPRY1/2) $(27,28)$. Besides, some other targets are also suggested that contain miR-21 specific binding sites, including peroxisome proliferator-activated receptor

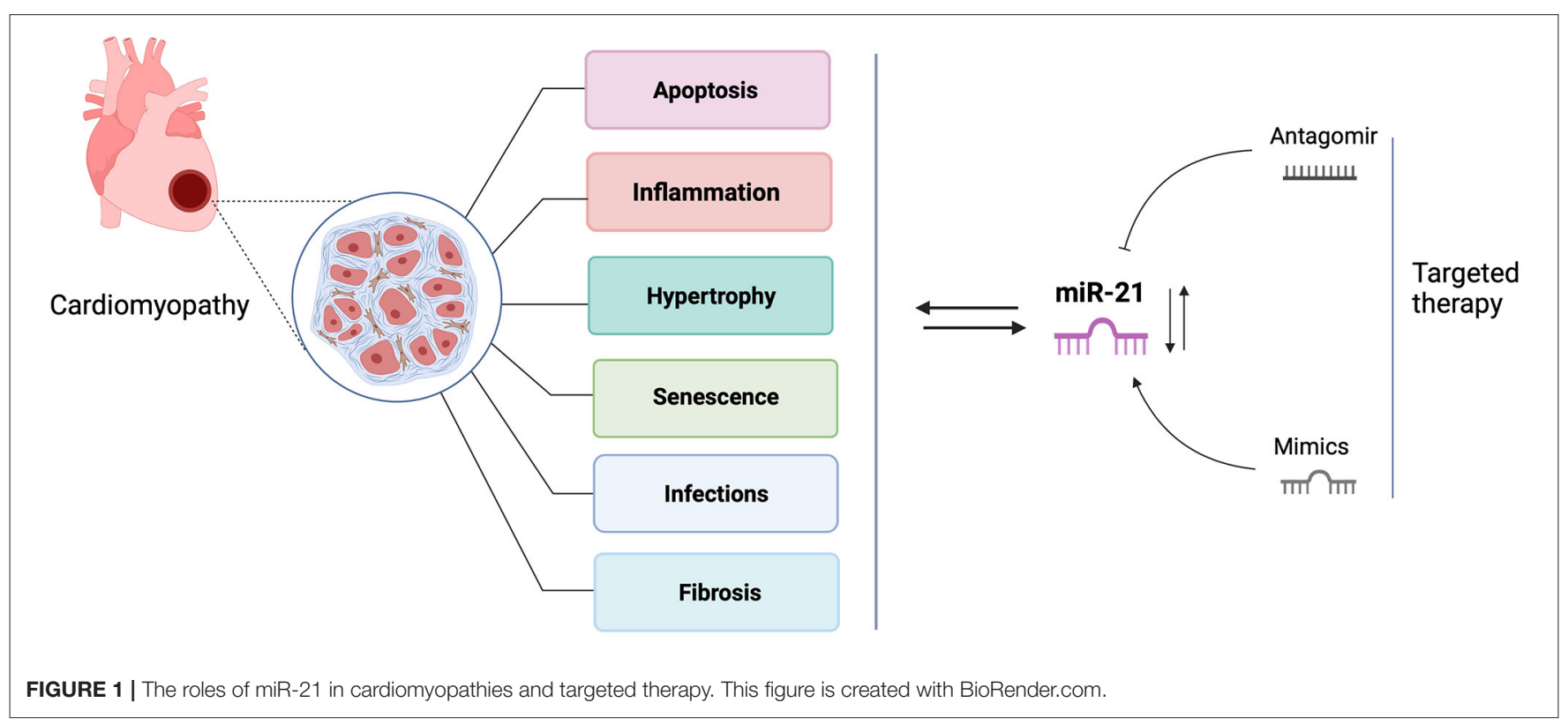


TABLE 1 | miR-21 expression pattern in cardiomyopathies.

\begin{tabular}{|c|c|c|c|}
\hline Disease & Animal study & Clinical study & Biomarker \\
\hline Ml & $\begin{array}{l}\text { Rat Heart \# (33) } \\
\text { Mouse Heart* }(34) \\
\text { Rat Heart } \uparrow(31,35) \\
\text { Mouse Heart } \downarrow(36)\end{array}$ & $\begin{array}{l}\text { Plasma* (37) } \\
\text { Serum } \uparrow(38)\end{array}$ & $\checkmark$ \\
\hline CAD & Mouse Plasma个(39) & $\begin{array}{l}\text { Plasma } \uparrow(6,39-41) \\
\text { PBMCs } \downarrow \text { (42) }\end{array}$ & $\checkmark$ \\
\hline AF & Rabbit Heart $\uparrow(43)$ & $\begin{array}{l}\text { Left atria } \uparrow(44) \\
\text { Myocytes } \uparrow(45) \\
\text { Plasma } \downarrow \text { (46) } \\
\text { Serum } \downarrow(47)\end{array}$ & $\checkmark$ \\
\hline $\mathrm{HF}$ & & $\begin{array}{l}\text { Plasma } \uparrow(48) \\
\text { Serum } \downarrow \text { (49) } \\
\text { Plasma* }^{*}(50)\end{array}$ & $\checkmark$ \\
\hline DCM & $\begin{array}{l}\text { Mouse Heart } \uparrow(26,51) \\
\text { Mouse Heart } \downarrow(52)\end{array}$ & $\begin{array}{l}\text { Heart } \uparrow(53) \\
\text { Serum } \uparrow(54)\end{array}$ & $\checkmark$ \\
\hline I/R Injury & $\begin{array}{l}\text { Mouse Heart } \uparrow(55,56) \\
\text { Rat Heart } \downarrow(57)\end{array}$ & Heart $\uparrow(58)$ & $\checkmark$ \\
\hline
\end{tabular}

Myocardial infraction (MI), Coronary artery disease (CAD), Atrial Fibrillation (AF), Heart Failure (HF), Diabetic cardiomyopathy (DCM), Ischemia-Reperfusion injury (I/R injury), Time-dependent change ${ }^{*}$, Site-dependent change ${ }^{\#}$, Increase $\uparrow$, Decrease $\downarrow$, Suggested as a biomarker $\checkmark$.

alpha(PPAR- $\alpha$ ) (29), a-kinase anchoring protein 8 (Akap8), and BRCA1 associated RING domain 1 (Bard1) (30), Jagged 1 (31), sorbin and SH3 domain-containing protein 2 (SORBS2) and PDZ and LIM domain 5 (PDLIM5) (32).

\section{EXPRESSION PATTERNS OF miR-21 IN CARDIOMYOPATHY}

The expression pattern of miR-21 in cardiovascular diseases has been extensively studied in recent years. Previous studies imply that there is a correlation between miR-21 expression level and cardiac diseases. Therefore, many studies were carried out to verify whether or not miR-21 can be a predictive or prognostic marker of heart diseases. Here we focus on the contradictory expression pattern of miR-21 in heart diseases (Table 1).

\section{Vitro Study and Animal Model Study}

In vitro studies, two main cell types, cardiomyocytes and cardiac fibroblasts are used to illustrate the miR-21 expression pattern and function.

In most cases, miR-21 expression levels were elevated in stress conditions such as oxidative stress, high glucose, and hypoxia (59-62). However, oxygen-glucose deprivation (OGD) and palmitate exposure can considerably decrease miR-21 expression levels in cardiomyocytes $(63,64)$.

Furthermore, murine models were applied to study miR-21 expression profiles in cardiomyopathy showing different miR21 responses to cardiac damage. miR-21 was down-regulated in infarcted areas but was upregulated in border areas in the acute myocardial infarction (AMI) rat model (33). In the myocardial infarction (MI) mouse model miR-21 expression changed in a time-dependent manner, increased in the first and second week but unchanged in the fourth week (34). Besides, two independent studies in mice models showed opposite miR-21 expression trends. One was observed an increase of miR-21 in the infarcted zone after MI, and the other showed a decrease of miR-21 after AMI $(35,36)$. Similarly, the reverse expression trends were also observed in ischemia/reperfusion (I/R) murine injury models. In the I/R injury mouse model, miR-21 was upregulated in the heart $(55,56)$, whilst heart miR-21 expression level decreased in the myocardial I/R injury rat model (57).

\section{Clinical Study}

In the clinical studies, the expression level of miR-21 was measured in the blood and heart tissue of patients suffering from different heart diseases. The results showed divergent expression trends. In the case of atrial fibrillation (AF), it is suggested that miR-21 might be involved in the pathogenesis of atrial fibrosis due to its increased expression in left atrial tissue and positive correlation with atrial collagen content (44). Another study also showed that miR-21 was highly expressed in human atrial myocytes from patients with chronic atrial fibrillation compared to the sinus rhythm group (45). By contrast, different independent studies observed significantly decreased miR-21 levels in plasma or serum in AF patients $(46,47)$. Likewise, the reverse trends of miR-21 expression were also found in patients with coronary artery diseases. miR-21 expression levels were upregulated in plasma $(6,39-41)$, but dramatically decreased in peripheral blood mononuclear cells (PBMCs) (42).

In heart failure, for example, the upward trend expression of miR-21 was observed in circulation (48), but there was a decrease of miR-21 expression in serum in symptomatic heart failure patients (49). More surprisingly, patients with acute heart failure showed increased plasma miR-21 levels between admission and discharge of hospital, then decreased following clinical compensation period (50). Further, this time-dependent expression of circulating miR-21 occurred in patients with postmyocardial infarction as well. miR-21 decreased in the first 2 days and increased on the 5th day, then returned to the control level in the latter point of post-myocardial infarction (37). In another study, upregulated serum miR-21 expression was observed in elderly patients with acute myocardial infarction (AMI) (38).

In other cases, various cardiomyopathies, including dilated cardiomyopathy, left ventricular ejection fraction, and ischemic cardiomyopathy, upregulated miR-21 expression levels were shown in heart tissue and circulation $(53,54,58,65)$. In addition, increased miR-21 level was suggested as a new biomarker of cardiovascular disease-related premature death to predict patients at great risk of adverse outcomes $(66,67)$. However, unchanged expression miR-21 level was also reported in patients with hypertrophic cardiomyopathy (53).

In short, it has been shown that the distinct miR-21 expression pattern in one cardiac disease depending on different sample sources. Moreover, inconsistent trends were also observed from the same source of samples (such as plasma) in the same cardiac diseases. 
TABLE 2 | miR-21 functions in cardiomyopathies.

\begin{tabular}{|c|c|c|c|c|}
\hline Disease conditions & Sample sources & Targets & Functions & References \\
\hline $\mathrm{Ml}$ & Heart/CMs/MPs & PDCD4 & $\begin{array}{l}\text { Apoptosis } \downarrow \\
\text { Inflammation } \downarrow\end{array}$ & $(33,34,36,68)$ \\
\hline $\mathrm{HF}$ & Heart & PTEN & Angiogenesis/CMs Survival $\uparrow$ & (69) \\
\hline DCM & Heart, CMs & Gelsolin & Hypertrophy $\downarrow$ & (52) \\
\hline I/R Injury & Heart, CMs & PDCD4/PTEN & Apoptosis $\downarrow$ & $(56,70)$ \\
\hline Valve Replacement & Heart & & Apoptosis $\downarrow$ & (65) \\
\hline VMCs & Heart & MAP2K3 & Necrosis/ viral titers $\downarrow$ & $(71)$ \\
\hline $\mathrm{HR}$ & CMs & $\begin{array}{l}\text { PTEN/PDCD4 } \\
\text { Akap8/ Bard1 }\end{array}$ & Apoptosis $\downarrow$ & $(30,61,70)$ \\
\hline $\mathrm{OGD} / \mathrm{H}_{2} \mathrm{O}_{2}$ / Palmitate & $\begin{array}{l}\text { NRCMs/ CPCs } \\
\text { H9C2/ CSCs }\end{array}$ & PDCD4 & Apoptosis $\downarrow$ & $(36,60,64,70,72)$ \\
\hline $\mathrm{Ml}$ & Heart/CFs & Smad7/Jagged1/ Spry1 & Fibrosis $\uparrow$ & $(31,35,73)$ \\
\hline HF & CFs & Spry1 & Fibrosis $\uparrow$ & $(74)$ \\
\hline DCM & Heart & SPRY1 & Fibrosis $\uparrow$ & $(51)$ \\
\hline I/R injury & CFs & PTEN & Fibrosis $\uparrow$ & $(55)$ \\
\hline $\mathrm{HHD}$ & Heart & PDCD4 & Fibrosis $\uparrow$ & (75) \\
\hline $\mathrm{AF}$ & Herat/CFs/CMs & Spry1/Smad7 & Fibrosis/ arrhythmia $\uparrow$ & $(43-45)$ \\
\hline CAD & Coronary artery & & Plaque instability $\uparrow$ & $(41)$ \\
\hline$H G$ & CFs & DUSP8 & Fibrosis $\uparrow$ & $(51,59)$ \\
\hline Ang-II & CPCs/NRCMs & PDCD4 & Fibrosis/hypertrophy $\uparrow$ & $(62,75)$ \\
\hline
\end{tabular}

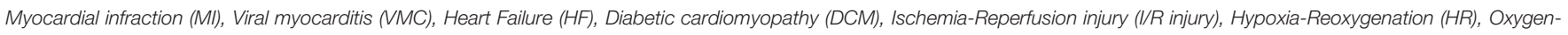

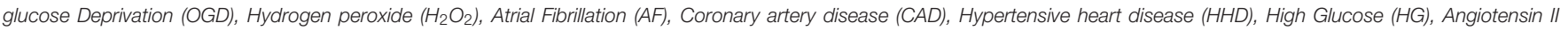

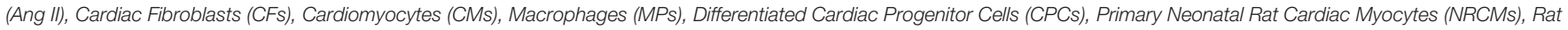

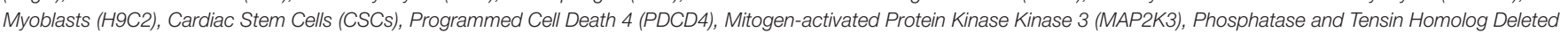

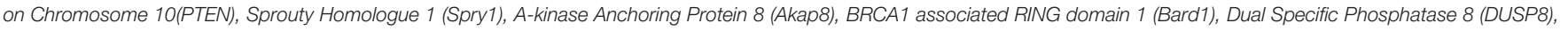
SMAD Family Member 7 (Smad 7), Increase $\uparrow$, Decrease $\downarrow$.

\section{FUNCTIONS OF MIR-21 IN CARDIAC DISEASES}

There is no doubt that miR-21 is a crucial regulator of developing cardiac diseases and the healing process after heart injury. However, it plays an important but controversial role. For instance, overexpression of miR-21 reduced myocardial infarct size by $29 \%$ at $24 \mathrm{~h}$ and decreased the dimension of left ventricles at 2 weeks after acute myocardial infarction (AMI). At the same time, miR-21 rescued cardiomyocytes from ischemia-induced cell apoptosis by targeting programmed cell death 4 (PDCD4) and activator protein 1(AP1) (33). On the contrary, miR-21 expression increased in the myocardial infarction mouse model, and transfection of miR-21 mimics into cardiac fibroblasts (CFs) caused activation of cardiac fibroblasts promoted cardiac fibrosis (35). Here, we discuss the diverse functions of miR-21 (Table 2) and the underlying cellular and molecular mechanism in cardiomyopathy (Figure 2).

\section{Protective Roles}

Positive functions of miR-21 were studied in a range of heart diseases by both gains-of-function and loss-of-function. In myocardial infarction (MI), infarct heart size was decreased, and heart function improved after transfection of miR21 extracellular vesicles (EVs) or lentivirus overexpressing miR-21. The further mechanism study revealed that miR-21 downregulated programmed cell death 4 (PDCD4) and apoptosis markers in cardiomyocytes, including bcl-2-like protein 4 (bax) and caspase-3 $(34,36)$. The similar protective effects of miR-21 also showed in myocardial ischemia-reperfusion (IR), diabetic cardiomyopathy, and hypertrophic cardiomyopathy. miR-21 attenuates cardiac injury and promotes cardiac myocytes survival through targeting PDCD4, gelsolin, and well-known tumor suppressor gene PTEN (52, 56, 70). Apart from antiapoptotic function, miR-21 plays an anti-inflammatory role in cardiomyopathy as well. miR-21 mimics notably decreased the rate of tumor necrosis factor (TNF)- $\alpha$-induced apoptosis in human cardiomyocytes by activating the JNK/p38/caspase3 signaling pathway (38). In the $\mathrm{I} / \mathrm{R}$ injury study, the overexpression of miR-21 effectively inhibited the TLR4/NF$\kappa \mathrm{B}$ pathway that reduced the level of myocardial apoptosis and inhibited the release of pro-inflammatory factors (57). Furthermore, a study has presented a new targeted therapy that miR-21 nanoparticle delivery into cardiac macrophages could promote angiogenesis, reduce hypertrophy, fibrosis, and cell apoptosis in the remote myocardium (68). miR-21 also can promote the proliferation of cardiac stem cells (CSCs). miR21 up-regulation with miR-21 mimics efficiently accelerated cell viability and proliferation in CSCs and protected them from oxidative injury by activating PTEN/PI3K/Akt pathway $(72,76)$. It is also proven that miR-21 can decrease cell apoptosis, inhibit viral progeny release in the heart, and protect it from coxsackievirus B3 infection through MAP2K3/P38 MAPK signaling pathway (77). Overall, miR-21 has beneficial effects on diseased cardiac tissues mainly by inhibiting PTEN and PDCD4 and promoting cell survival and proliferation. 


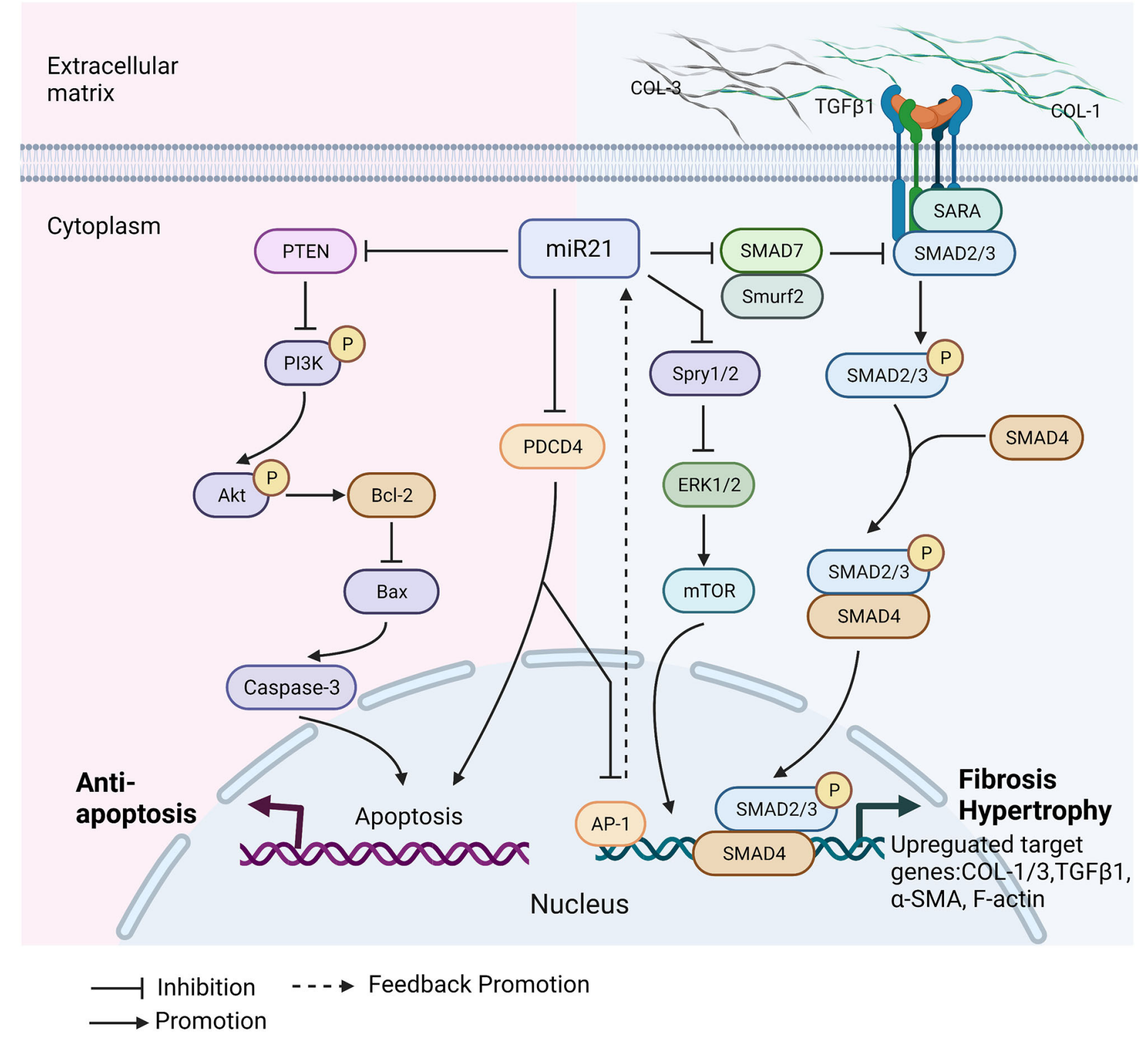

FIGURE 2 | Molecular mechanisms of miR-21 involvement in cardiomyopathies. Cardiomyopathy alters miR-21 expression level in heart and circulation. miR-21 has protective effects on heart in cardiomyopathy. It regulates cardiac cell death by targeting PTEN and PDCD4. There is a positive promotion loop between miR-21 and AP-1. Overexpression of miR-21 inhibits PDCD4, and further increases AP-1, which is a transcription factor directly promotes miR-21 expression. PTEN downregulation activates PI3K/Akt pathway to promote cardiomyocytes survival and proliferation, that can protect against heart dysfunction. In the meantime, miR-21 has also negative effects on cardiac diseases' development. miR-21 regulates smad7/smad2/3 and Spry/ERK pathways to promote cardiac fibrosis by increasing collagens deposition, TGF $\beta 1, \alpha$-smooth muscle actin ( $\alpha$-SMA) and filamentous actin (F-actin) polymerization. Spry/ERK/mTOR pathway contributes to myocardial hypertrophy through increasing myofibroblast survival. Collagen type I/III (COL1/3), PTEN, Phosphoinositide 3-kinases (PI3K), Protein kinase B(Akt), B-cell lymphoma 2 (Bcl-2), bcl-2-like protein 4 (Bax), Programmed Cell Death 4 (PDCD4), Activator protein 1(AP-1), Extracellular signal-regulated kinase (ERK), Sprouty 1/2(Spry 1/2), Mammalian target of rapamycin (mTOR), SMAD Specific E3 Ubiquitin Protein Ligase 2 (Smurf 2), Smad anchor for receptor activation (SARA), SMAD Family Member 2/3/4/7 (Smad2/3/4/7), Filamentous actin (F-actin), Alpha smooth muscle actin ( $\alpha$-SMA). This figure is created with BioRender.com.

\section{Detrimental Roles Cardiac Fibrosis}

Cardiac fibrosis is characterized by excessive and continuous collagens deposition and activation of cardiac fibroblasts and differentiation to myofibroblasts $(69,78)$. Various conditions can promote cardiac fibrosis, such as myocardial infarction, hypertension, and diabetic cardiomyopathy (79). Despite cardiac fibrosis is a self-healing process, it also leads to severe cardiac dysfunctions due to abnormal extracellular matrix (ECM) remodeling (80). In the significant number of studies, it has been demonstrated that miR-21 is involved in cardiac fibrosis $(54,75)$. miR-21 directly regulates downstream 
targets and engages in a variety of fibrotic activities that include increasing transforming growth factor $\beta$ 1(TGF $\beta-1)$, cardiac fibroblasts activation, and endothelial-mesenchymal transition (EndMT) (26). Here, we summarize miR-21mediated two main cardiac fibrosis signaling pathways in cardiomyopathies:

\section{(i) $\mathrm{miR}-21 / \mathrm{TGF}-\beta 1 / \mathrm{smad} 7$ Pathway}

Cardiac fibrosis is tightly dependent on transforming growth factor beta-1 (TGF- $\beta 1$ ), contributing to cardiac fibroblast differentiation to myofibroblast $(81,82)$. In the diseased condition, miR-21 and TGF- $\beta 1$ expression levels are commonly upregulated in cardiac fibroblasts. Functionally, there is a mutual promotion loop between miR-21 and TGF- $\beta 1$ : transfection of miR-21 mimics into cardiac fibroblasts increases TGF- $\beta 1$ synthesis and secretion, and similarly, cardiac fibroblasts treat with TGF- $\beta 1$ boost miR-21 expression (63). Studies suggest that Smad7 is an essential direct target gene of miR-21 and a critical negative regulator in this feedback loop between miR-21 and TGF- $\beta 1$ (43). Smad7 is capable of inhibiting TGF- $\beta 1$ signaling by preventing the phosphorylation of $S m a d 2 / 3$ or recruits the ubiquitin ligases Smurf1 and Smurf2 to induce proteasomal degradation of the receptor complexes $(26,83)$. miR-21 inhibition upregulated Smad7 expression, significantly alleviated myocardial fibrosis in vivo, and prevented cardiac fibroblasts activation in vitro study (35).

(ii) miR-21/ TGF- $\beta 1 /$ spry1 Pathway

The Sprouty (Spry) family is first identified as a negative regulator of fibroblast growth factor (FGF) in Drosophila (73). Spry1 belongs to the Sprouty (Spry) family, an evolutionally conserved gene that acts as a potent inhibitor of the Ras/MEK/ERK pathway (84). Spry1 is a target of miR-21. The 3' untranslated region (UTR) of Spry1 mRNA contains several predicted microRNA-binding sites, of which only miR-21 levels are increased selectively in fibroblasts of the failing heart. Increased miR-21 blocks Spry1 and occurs further Inhibition of ERKMAP kinase activity (74). This mechanism regulates fibroblast survival and growth factor secretion, TGF- $\beta 1$ in particular. When TGF- $\beta 1$ is over-expressed, that leads to downregulate the expression level of Spry1, thereby controlling the extent of interstitial fibrosis and cardiac hypertrophy (85). A study showed that miR-21 was highly expressed in left atria from patients with atrial fibrillation, which positively correlated with collagen content and caused a reduction of protein expression of Spry1 and increased connective tissue growth factor (CTGF), lysyl oxidase, and Rac1-GTPase (44). Likewise, another study demonstrated that miR-21 promoted postmyocardial infraction fibrosis by targeting Spry1, which inhibits ERK1/2 activation and mediates TGF- $\beta / S m a d 2 / 3$ pathway activation (73). Besides, overexpression of miR-21 induced myocardial fibrosis by regulating two other targets: Jagged 1 and dual specific phosphatase 8 (DUSP8). miR-21 suppressed DUSP8 expression and promoted high glucose-induced cardiac fibrosis through the JNK/SAPK and p38 signaling pathways $(31,59)$.

\section{Cardiac Hypertrophy}

In diabetic cardiomyopathy (DM), miR-21 knockout mice showed less severe cardiac hypertrophy and cardiac dysfunction than wide-type DM mice. Overexpression of miR-21 aggravated fibrosis, reduced autophagy through the miR-21/SPRY1/ERK/mTOR pathway (51).

\section{Cardiac Senescence and Inflammaging}

Inflammaging is a major risk factor for heart failure and overall cardiac disorders, but the underlying molecular mechanism remains unclear. It has been suggested that miR-21 is involved in cardiac senescence and inflammaging. Circulating miR-21 was higher in elderly patients with the cardiovascular disease than in the age-matched healthy control group, and miR21 was correlated positively with inflammatory factors such as C-reactive protein and fibrinogen levels (86). In addition, another study showed that miR-21 was elevated in both aged myocardium and D-galactose-induced pseudo-aging mouse model, and further investigation showed that miR-21 promoted doxorubicin-induced cardiomyocyte senescence in vitro (87).

\section{Viral or Bacterial Infection-Induced Myocarditis}

miR-21 is one of the microRNAs that takes part in inflammatory pathogenesis in viral and bacterial myocarditis. Highly expressed miR-21-3p was observed in mouse heart challenged with lipopolysaccharide (LPS) and positively correlated with cardiac dysfunction. Meanwhile, the study suggested SORBS2 was a target gene of miR-21-3p (88). In Viral myocarditis, miR-21 was upregulated, and silencing miR-21 ameliorated viral myocarditis by blocking Th17 differentiation (71).

In brief, the conflicting data shows contradictory functions of miR-21 in cardiomyopathies. Indeed, the tasks of miR-21 in vivo studies are mysterious. Every research is unique, studying different cardiac diseases, even though looking at the same condition still differs in animal models or the progression timepoint of diseases. It is hard to find identical repeated studies to make the comparison among results.

However, the results of Vitro studies show that high consistency depends on specific cell types. If the studied subject is cardiomyocytes, the results indicate miR-21 has a protective effect on the heart, such as anti-apoptotic effect on cardiomyocytes; if the research subject is changed to cardiac fibroblasts, the results lead to demonstrate a negative role of miR21 in myocardial fibrosis. Intriguingly, neither Vivo studies nor Vitro studies try to illustrate both effects of miR-21 at the same time in one cardiac disease. Therefore, delicate and sophisticated research studies require to show miR-21 distinct functions in different cell types in vivo.

\section{CLINICAL IMPLICATIONS OF miR-21}

\section{Biomarker}

miR-21 has been proposed as a biomarker in a variety of heart diseases. A clinical biomarker is defined as "an indicator of normal biological processes, pathogenesis, or responses to an exposure or therapeutic intervention" (89). For instance, troponin $\mathrm{I}(\mathrm{cTnI})$ and $\mathrm{T}(\mathrm{cTnT})$ are unique heart proteins and are 
specific and sensitive biomarkers of myocardial injury in various cardiovascular disorders (90). Ideal biomarkers have the potential to provide valuable information that aids the diagnosis of a disease and predicts disease progression and outcomes. Bradford Hill's Guidelines can be applied to establish and evaluate prebiomarkers (91). Here, we list the first three criteria to show whether or not miR-21 can be a biomarker of cardiomyopathies.

The first essential requirement is that there should be strong associations between a biomarker and the outcome of a clinical disorder. Undoubtedly, miR-21 regulates key pathways and tightly relates to cardiac pathogenesis, such as cardiac fibrosis. Expression changes of miR-21 were observed in many studies, and the pathological roles were demonstrated by both gain- and loss- of-function study.

Additionally, consistent findings should be observed by different individuals in different places with different samples. miR-21 is not fulfilled with this point. The inconsistent expression levels and contradictory functions have been reported throughout the last two decades. miR-21 expression levels in circulation and heart tissue were various in independent studies. A plausible reason is that there are no standard reference values for miR-21 expression in physiological and pathological conditions. The expression levels in control groups might vary from subject to subject.

Most importantly, an ideal marker is supposed to express at a specific site and associate with a particular disease. miR21 is not a specific miRNA in the heart. miR-21 is expressed abundantly and ubiquitously in some other multiple types of tissues, such as the liver, kidney, nervous system, and immune cells (92). Not only does diseased heart tissue contribute to the circulation level of miR-21, but other tissues, especially those globally involved in various diseases, namely immune cells, also secret miR-21 to respond to pathological stimuli via inflammation pathways (93). In addition, miR-21 is not a diseasespecific marker either. miR-21 is suggested as a marker in various cardiovascular diseases, including heart failure, atrial fibrillation, and dilated cardiomyopathy. Apart from cardiovascular diseases, numerous studies implicated miR-21 as a biomarker in many other diseases ranging from cancer, renal fibrosis, and diabetes (94). So far, miR-21 cannot be considered an ideal biomarker for a specific cardiomyopathy.

\section{Targeted Therapy}

With increasing interest in miRNAs, miRNA-based drug development is already in progress. Over the last decade, several biopharmaceutical industries have launched miRNA projects in their development pipeline. For example, the first miRNAtargeted drug for treating hepatitis $\mathrm{C}$ virus (HCV) infection sponsored by Santaris Pharma in 2012 is miravirsen. It is an LNA-modified antimiR oligonucleotide, targets miR122 (95). miRNA-based drugs have two main types: AntagomiRs, which can specifically bind to target miRNAs in a complementary fashion and inhibit their functions; another is synthetic miRNA mimics, which can restore the reduced expression of miRNAs in disease conditions (96). Currently, there are going on many miR-21-based diagnostics and therapeutic clinical trials in a range of diseases, such as Predictive and Prognostic Value of Inflammatory Markers in Stage IV
Colorectal Cancer (NCT04149613), miR-200b \& miR-21 in Diabetic Wounds recruiting (NCT02581098), and Study of Lademirsen (SAR339375) in Patients with Alport Syndrome (HERA) (NCT02855268). Of note, miRNA-based drugs are currently not available for diagnostics and therapeutic purposes.

Similarly, miR-21 has emerged as a potential therapeutic target in cardiomyopathy due to its participation in the pathogenesis of many cardiac diseases via regulating cardiomyocytes apoptosis and cardiac fibrosis. Numerous preclinical studies have been carried out to illustrate its therapeutic outcomes in cardiomyopathies. For instance, a study demonstrated that miR-21 overexpression by nucleofection in human cardiac fibroblasts with the expression vector leads to upregulation of collagen expression. In contrast, miR-21 Inhibition by LNA anti-miR-21 reduced collagen expression and alleviated cardiac fibrosis (82).

However, developing miR-21 targeted treatment for cardiomyopathies has several challenges. Firstly, It is challenging to apply repression or restoration, because the confusion of miR-21 expression pattern in cardiomyopathies. Besides, it is problematic for targeted therapy that miR-21 is not an organ or tissue-specific miRNA, not even cell type-specific miRNA. Hence, issues with off-target effects and undesired on-target effects have to be taken into account (97).

Despite the considerable understanding of miR-21 regulation, further studies require to obtain sufficient knowledge of miR21 in its spatial and temporal regulation. miR-21 expression is tightly regulated by multiple factors. Several promotors can effectively induce miR-21 expression at the transcriptional level, including AP-1, STAT3, Ras, ERK1/2, and EGFR (98-102). Oppositely, some transcriptional suppressors have also been reported. For instance, miR-21 transcription was repressed by NFI, C/EBP, Gfil, and estrogen receptor $(98,103,104)$. Moreover, miR-21 expression is regulated at the post-translational level. TGF $\beta$ and BMP4 (a member of the TGF $\beta$ superfamily) upregulate pre-mir-21 expression and SMADs (SMAD1/5 and SMAD2/3) mediate pri-mir-21 expression (105). On the contrary, BMP6 (a member of the TGF $\beta$ superfamily) has been shown to inhibit miR-21 expression (106). In addition, miR-21 expression can be modulated by other noncoding RNA associated with various molecular pathways. Tumor-Associated Long ncRNA Expressed on Chromosome 2(TALNEC2) was highly expressed in myocardial ischemic patients. Overexpression of TALNEC2 downregulated miR-21 and aggravated hypoxic injury by miR-21/PDCD4-mediated activation of the $\mathrm{Wnt} / \beta$-catenin signaling pathway in myocardial ischemic injury (107). In cardiovascular diseases, LncRNA MEG3 is downregulated and negatively correlates with miR21 levels. MEG3 overexpression suppresses endothelial cell proliferation and migration and reduces proteoglycan, type $\mathrm{I}$, and $\mathrm{V}$ collagen expression through the decrease of miR21 (108).

\section{SUMMARY AND PERSPECTIVE}

The heart is an important and complex organ. Many diseases can affect the functionality of the heart to cause a range of complex conditions. Cardiomyopathy is defined as a heart 
muscle disease, but not only cardiomyocytes and some other heart cells are also involved in the occurrence and progression of cardiomyopathy. The cardiac muscle is composed of various cell types. Cardiomyocytes account for $25-35 \%$ of all cells in the adult cardiac muscle, and other heart cell types make up 65$75 \%$, including endothelial cells, smooth muscle cells, fibroblasts, and immune cells (109). Both cardiomyocytes and noncardiomyocytes respond to physiological and pathological stress, such as cardiomyocytes apoptosis, cardiac fibroblast activation, and immune cell infiltration. Those all play indispensable roles in heart pathogenesis (110). miRNAs expression profiles may be completely distinct based on different heart cell types $(111,112)$. Similarly, miR-21 may vary in expression signatures and functions in different heart cells. As reported, miR-21 overexpression activates fibroblast to trigger the fibrosis process but stimulates proliferation of cardiomyocytes against stress overloaded conditions. The detailed expression map of miR-21 remains elusive, so that it is challenging to apply miR-21 as a biomarker in heart disease diagnosis or prognosis. Moreover, the biology of miR-21 has not been fully clarified. It is not clear that the regulation details in miR-21 biogenesis, secretion, and degradation. Thus, further studies are necessary to understand the molecular basis of distinct functions and identify specific target genes of miR-21 in different tissues. Furthermore, it is not only miR-21, the multiple miRNAs involved in heart diseases. It has been reported that dozens of miRNAs are associated with heart failure, myocardial infarction, and arrhythmia (113).

\section{REFERENCES}

1. Schaufelberger M. Cardiomyopathy and pregnancy. Heart. (2019)105:15431551. doi: $10.1136 /$ heartjnl-2018-313476

2. Bartel DP. Metazoan microRNAs. Cell. (2018)173:2051. doi: 10.1016/j.cell.2018.03.006

3. Cordes KR, Srivastava D. MicroRNA regulation of cardiovascular development. Circ Res. (2009)104:72432. doi: 10.1161/CIRCRESAHA.108.192872

4. Kan C, Cao J, Hou J, Jing X, Zhu Y, Zhang J, et al. Correlation of miR-21 and BNP with pregnancy-induced hypertension complicated with heart failure and the diagnostic value. Exp Ther Med. (2019)17:312935. doi: 10.3892/etm.2019.7286

5. Zhang Y, Liu YJ, Liu T, Zhang H, Yang SJ. Plasma microRNA-21 is a potential diagnostic biomarker of acute myocardial infarction. Eur Rev Med Pharmacol Sci. (2016)20:323-9.

6. Kumar D, Narang R, Sreenivas V, Rastogi V, Bhatia J, Saluja D, et al. Circulatory miR-133b and miR-21 as novel biomarkers in early prediction and diagnosis of coronary artery disease. Genes. (2020) 11:164. doi: 10.3390/genes11020164

7. Tao L, Huang X, Xu M, Qin Z, Zhang F, Hua F, et al. Value of circulating miRNA-21 in the diagnosis of subclinical diabetic cardiomyopathy. Mol Cell Endocrinol. (2020) 518:110944. doi: 10.1016/j.mce.2020. 110944

8. Yang W, Shao J, Bai X, Zhang G. Expression of plasma microRNA$1 / 21 / 208 \mathrm{a} / 499$ in myocardial ischemic reperfusion injury. Cardiology. (2015) 130:237-41. doi: 10.1159/000371792

9. Cheng Y, Zhang C. MicroRNA-21 in cardiovascular disease. J Cardiovasc Transl Res. (2010) 3:251-5. doi: 10.1007/s12265-010-9169-7

10. Dai B, Wang F, Nie X, Du H, Zhao Y, Yin Z, et al. The cell typespecific functions of miR-21 in cardiovascular diseases. Front Genet. (2020) 11:563166. doi: 10.3389/fgene.2020.563166
Studies show that miR-1, miR133a, miR-208a/b, and miR-499 have explicitly enriched the myocardium and might be specific therapeutic targets in cardiomyopathy (114). For example, miR499 participates in the development of myocardial hypertrophy and fibrosis by targeting many intracellular signaling molecules and transcription factors, including Akt, mitogen-activated protein kinases (MAPKs), and early growth response 1/2 (Egr1/2) (115). Besides, miR-21 may interact with other microRNAs that participate in cardiac pathogenesis. A cancer study demonstrated a high level of miR-21 increased miR-499 expression level by stabilizing mature miR-499 and affecting its turnover rate, resulting in the suppression of PDCD4 in head and neck squamous cell carcinoma (HNSCC) (116).

Nonetheless, miR-21 studies shed light on the understanding of cellular and molecular mechanisms of cardiomyopathy and offered a potential therapeutic target for clinical intervention.

\section{AUTHOR CONTRIBUTIONS}

S, GP, and MB: concept and design. S, RF, and LS: drafting of the manuscript. All authors have read and agreed to the published version of the manuscript.

\section{FUNDING}

This review was supported by the Ministero dell'Istruzione, dell'Università e della Ricerca Scientifica (Grants PRIN 2017).
11. Patrick DM, Montgomery RL, Qi X, Obad S, Kauppinen S, Hill JA, et al. Stress-dependent cardiac remodeling occurs in the absence of microRNA-21 in mice. J Clin Invest. (2010) 120:3912-6. doi: 10.1172/JCI43604

12. Zeng Y, Cullen BR. Sequence requirements for micro RNA processing and function in human cells. RNA. (2003) 9:112-23. doi: 10.1261/rna.2780503

13. Lee RC, Feinbaum RL, Ambros V. The C. elegans heterochronic gene lin-4 encodes small RNAs with antisense complementarity to lin-14. Cell. (1993) 75:843-54. doi: 10.1016/0092-8674(93)90529-Y

14. Hammond SM. An overview of microRNAs. Adv Drug Deliv Rev. (2015) 87:3-14. doi: 10.1016/j.addr.2015.05.001

15. Krzywinska O, Bracha M, Jeanniere C, Recchia E, Kedziora Kornatowska K, Kozakiewicz M. Meta-analysis of the potential role of miRNA-21 in cardiovascular system function monitoring. Biomed Res Int. (2020) 2020:4525410. doi: 10.1155/2020/4525410

16. Cai X, Hagedorn $\mathrm{CH}$, Cullen BR. Human microRNAs are processed from capped, polyadenylated transcripts that can also function as mRNAs. RNA. (2004) 10:1957-66. doi: 10.1261/rna.7135204

17. Lee Y, Kim M, Han J, Yeom KH, Lee S, Baek SH, et al. MicroRNA genes are transcribed by RNA polymerase II. EMBO J. (2004) 23:405160. doi: 10.1038/sj.emboj.7600385

18. Han J, Lee Y, Yeom KH, Nam JW, Heo I, Rhee JK, et al. Molecular basis for the recognition of primary microRNAs by the Drosha-DGCR8 complex. Cell. (2006) 125:887-901. doi: 10.1016/j.cell.2006.03.043

19. Yeom KH, Lee Y, Han J, Suh MR, Kim VN. Characterization of DGCR8/Pasha, the essential cofactor for Drosha in primary miRNA processing. Nucleic Acids Res. (2006) 34:4622-9. doi: 10.1093/nar/gkl458

20. Bohnsack MT, Czaplinski K, Gorlich D. Exportin 5 is a RanGTP-dependent dsRNA-binding protein that mediates nuclear export of pre-miRNAs. RNA. (2004) 10:185-91. doi: 10.1261/rna.5167604

21. Kawamata T, Seitz H, Tomari Y. Structural determinants of miRNAs for RISC loading and slicer-independent unwinding. Nat Struct Mol Biol. (2009) 16:953-60. doi: 10.1038/nsmb.1630 
22. Friedman RC, Farh KK, Burge CB, Bartel DP. Most mammalian mRNAs are conserved targets of microRNAs. Genome Res. (2009) 19:92105. doi: 10.1101/gr.082701.108

23. Condorelli G, Latronico MV, Dorn 2nd GW. microRNAs in heart disease: putative novel therapeutic targets? Eur Heart J. (2010)31:64958. doi: 10.1093/eurheartj/ehp573

24. Cheng Y, Liu X, Zhang S, Lin Y, Yang J, Zhang C. MicroRNA-21 protects against the $\mathrm{H}(2) \mathrm{O}(2)$-induced injury on cardiac myocytes via its target gene PDCD4. J Mol Cell Cardiol. (2009) 47:5-14. doi: 10.1016/j.yjmcc.2009.01.008

25. Liu Z, Wang H, Hou G, Cao H, Zhao Y, Yang B. Notoginsenoside R1 protects oxygen and glucose deprivation-induced injury by upregulation of miR-21 in cardiomyocytes. J Cell Biochem. (2019) 120:9181-92. doi: 10.1002/jcb.28194

26. Li Q, Yao Y, Shi S, Zhou M, Zhou Y, Wang M, et al. Inhibition of miR-21 alleviated cardiac perivascular fibrosis via repressing EndMT in T1DM. J Cell Mol Med. (2020) 24:910-20. doi: 10.1111/jcmm.14800

27. Xu HF, Ding YJ, Zhang ZX, Wang ZF, Luo CL, Li BX, et al. MicroRNA21 regulation of the progression of viral myocarditis to dilated cardiomyopathy. Mol Med Rep. (2014) 10:161-8. doi: 10.3892/mmr.2014.2205

28. Sayed D, Rane S, Lypowy J, He M, Chen IY, Vashistha H, et al. MicroRNA21 targets Sprouty2 and promotes cellular outgrowths. Mol Biol Cell. (2008) 19:3272-82. doi: 10.1091/mbc.e08-02-0159

29. Chuppa S, Liang M, Liu P, Liu Y, Casati MC, Cowley AW, et al. MicroRNA21 regulates peroxisome proliferator-activated receptor alpha, a molecular mechanism of cardiac pathology in Cardiorenal Syndrome Type 4. Kidney Int. (2018) 93:375-89. doi: 10.1016/j.kint.2017.05.014

30. Shen H, Yao Z, Zhao W, Zhang Y, Yao C, Tong C. miR-21 enhances the protective effect of loperamide on rat cardiomyocytes against hypoxia/reoxygenation, reactive oxygen species production and apoptosis via regulating Akap8 and Bard1 expression. Exp Ther Med. (2019) 17:131220. doi: $10.3892 /$ etm.2018.7047

31. Zhou XL, Xu H, Liu ZB, Wu QC, Zhu RR, Liu JC. miR-21 promotes cardiac fibroblast-to-myofibroblast transformation and myocardial fibrosis by targeting Jagged1. J Cell Mol Med. (2018) 22:3816-24. doi: 10.1111/jcmm.13654

32. Bang C, Batkai S, Dangwal S, Gupta SK, Foinquinos A, Holzmann A, et al. Cardiac fibroblast-derived microRNA passenger strand-enriched exosomes mediate cardiomyocyte hypertrophy. J Clin Invest. (2014) 124:213646. doi: 10.1172/JCI70577

33. Dong S, Cheng Y, Yang J, Li J, Liu X, Wang X, et al. MicroRNA expression signature and the role of microRNA-21 in the early phase of acute myocardial infarction. J Biol Chem. (2009) 284:29514-25. doi: 10.1074/jbc.M109.027896

34. Gu GL, Xu XL, Sun XT, Zhang J, Guo CF, Wang CS, et al. Cardioprotective effect of MicroRNA-21 in murine myocardial infarction. Cardiovasc Ther. (2015) 33:109-17. doi: 10.1111/1755-5922.12118

35. Yuan J, Chen H, Ge D, Xu Y, Xu H, Yang Y, et al. Mir-21 promotes cardiac fibrosis after myocardial infarction via targeting Smad7. Cell Physiol Biochem. (2017) 42:2207-19. doi: 10.1159/000479995

36. Gu H, Liu Z, Li Y, Xie Y, Yao J, Zhu Y, et al. Serum-derived extracellular vesicles protect against acute myocardial infarction by regulating miR-21/PDCD4 signaling pathway. Front Physiol. (2018) 9:348. doi: 10.3389/fphys.2018.00348

37. Zile MR, Mehurg SM, Arroyo JE, Stroud RE, DeSantis SM, Spinale FG. Relationship between the temporal profile of plasma microRNA and left ventricular remodeling in patients after myocardial infarction. Circ Cardiovasc Genet. (2011) 4:614-9. doi: 10.1161/CIRCGENETICS.111.9 59841

38. Wang ZH, Sun XY, Li CL, Sun YM, Li J, Wang LF, et al. miRNA21 expression in the serum of elderly patients with acute myocardial infarction. Med Sci Monit. (2017) 23:5728-34. doi: 10.12659/MSM.9 04933

39. Han H, Qu G, Han C, Wang Y, Sun T, Li F, et al. MiR-34a, miR-21 and miR-23a as potential biomarkers for coronary artery disease: a pilot microarray study and confirmation in a 32 patient cohort. Exp Mol Med. (2015) 47:e138. doi: 10.1038/emm.2014.81

40. Jansen F, Schafer L, Wang H, Schmitz T, Flender A, Schueler R, et al. Kinetics of circulating MicroRNAs in response to cardiac stress in patients with coronary artery disease. J Am Heart Assoc. (2017) 6: e005270. doi: 10.1161/JAHA.116.005270
41. He W, Zhu L, Huang Y, Zhang Y, Shen W, Fang L, et al. The relationship of MicroRNA-21 and plaque stability in acute coronary syndrome. Medicine. (2019) 98:e18049. doi: 10.1097/MD.0000000000018049

42. Nariman-Saleh-Fam Z, Vahed SZ, Aghaee-Bakhtiari SH, Daraei A, Saadatian Z, Kafil HS, et al. Expression pattern of miR-21, miR-25 and PTEN in peripheral blood mononuclear cells of patients with significant or insignificant coronary stenosis. Gene. (2019) 698:1708. doi: 10.1016/j.gene.2019.02.074

43. He X, Zhang K, Gao X, Li L, Tan H, Chen J, et al. Rapid atrial pacing induces myocardial fibrosis by down-regulating Smad7 via microRNA-21 in rabbit. Heart Vessels. (2016) 31:1696-708. doi: 10.1007/s00380-016-0808-z

44. Adam O, Lohfelm B, Thum T, Gupta SK, Puhl SL, Schafers HJ, et al. Role of miR-21 in the pathogenesis of atrial fibrosis. Basic Res Cardiol. (2012) 107:278. doi: 10.1007/s00395-012-0278-0

45. Barana A, Matamoros M, Dolz-Gaiton P, Perez-Hernandez M, Amoros I, Nunez $M$, et al. Chronic atrial fibrillation increases microRNA-21 in human atrial myocytes decreasing L-type calcium current. Circ Arrhythm Electrophysiol. (2014) 7:861-8. doi: 10.1161/CIRCEP.114.001709

46. McManus DD, Tanriverdi K, Lin H, Esa N, Kinno M, Mandapati D, et al. Plasma microRNAs are associated with atrial fibrillation and change after catheter ablation (the miRhythm study). Heart Rhythm. (2015) 12:310. doi: 10.1016/j.hrthm.2014.09.050

47. Sieweke JT, Pfeffer TJ, Biber S, Chatterjee S, Weissenborn K, Grosse GM, et al. miR-21 and NT-proBNP correlate with echocardiographic parameters of atrial dysfunction and predict atrial fibrillation. J Clin Med. (2020) 9:1118. doi: $10.3390 / \mathrm{jcm} 9041118$

48. Ding $\mathrm{H}$, Wang $\mathrm{Y}$, Hu L, Xue S, Wang Y, Zhang L, et al. Combined detection of miR-21-5p, miR-30a-3p, miR-30a-5p, miR-155-5p, miR-216a and miR-217 for screening of early heart failure diseases. Biosci Rep. (2020) 40:BSR20191653. doi: 10.1042/BSR20191653

49. Tomaniak M, Sygitowicz G, Blaszczyk O, Koltowski L, Puchta D, Malesa $\mathrm{K}$, et al. miR-1, miR-21, and galectin-3 in hypertensive patients with symptomatic heart failure and left ventricular hypertrophy. Kardiol Pol. (2018) 76:1009-11. doi: 10.5603/KP.2018.0117

50. Schneider S, Silvello D, Martinelli NC, Garbin A, Biolo A, Clausell N, et al. Plasma levels of microRNA-21,-126 and-423-5p alter during clinical improvement and are associated with the prognosis of acute heart failure. Mol Med Rep. (2018) 17:4736-46. doi: 10.3892/mmr.2018.8428

51. Li X, Meng C, Han F, Yang J, Wang J, Zhu Y, et al. Vildagliptin attenuates myocardial dysfunction and restores autophagy via miR21/SPRY1/ERK in diabetic mice heart. Front Pharmacol. (2021) 12:634365. doi: 10.3389/fphar.2021.634365

52. Dai B, Li H, Fan J, Zhao Y, Yin Z, Nie X, et al. MiR-21 protected against diabetic cardiomyopathy induced diastolic dysfunction by targeting gelsolin. Cardiovasc Diabetol. (2018) 17:123. doi: 10.1186/s12933-018-0767-Z

53. Li M, Chen X, Chen L, Chen K, Zhou J, Song J. MiR-1-3p that correlates with left ventricular function of HCM can serve as a potential target and differentiate HCM from DCM. J Transl Med. (2018) 16:161. doi: 10.1186/s12967-018-1534-3

54. Rubis P, Toton-Zuranska J, Wisniowska-Smialek S, Holcman K, KoltonWroz M, Wolkow $\mathrm{P}$, et al. Relations between circulating microRNAs (miR21, miR-26, miR-29, miR-30 and miR-133a), extracellular matrix fibrosis and serum markers of fibrosis in dilated cardiomyopathy. Int J Cardiol. (2017) 231:201-6. doi: 10.1016/j.ijcard.2016.11.279

55. Roy S, Khanna S, Hussain SR, Biswas S, Azad A, Rink C, et al. MicroRNA expression in response to murine myocardial infarction: miR21 regulates fibroblast metalloprotease-2 via phosphatase and tensin homologue. Cardiovasc Res. (2009)82:21-9. doi: 10.1093/cvr/cvp015

56. Tu Y, Wan L, Fan Y, Wang K, Bu L, Huang T, et al. Ischemic postconditioning-mediated miRNA-21 protects against cardiac ischemia/reperfusion injury via PTEN/Akt pathway. PLoS ONE. (2013) 8:e75872. doi: 10.1371/journal.pone.0075872

57. Pan YQ, Li J, Li XW, Li YC, Li J, Lin JF. Effect of miR-21/TLR4/NFkappaB pathway on myocardial apoptosis in rats with myocardial ischemia-reperfusion. Eur Rev Med Pharmacol Sci. (2018) 22:792837. doi: 10.26355/eurrev_201811_16420

58. Kowara MK, Paskal W, Gondek A, Główczyńska R, Rybak K, Kubik M, et al. Increased plasma levels of hsa-miR-21-5p in patients with reduced 
left ventricle ejection fraction admitted urgently due to acute coronary syndrome. Med Res J. (2020) 5:250-5. doi: 10.5603/MRJ.a2020.0045

59. Liu S, Li W, Xu M, Huang H, Wang J, Chen X. Micro-RNA 21Targets dual specific phosphatase 8 to promote collagen synthesis in high glucosetreated primary cardiac fibroblasts. Can J Cardiol. (2014) 30:168999. doi: 10.1016/j.cjca.2014.07.747

60. Xiao J, Pan Y, Li XH, Yang XY, Feng YL, Tan HH, et al. Cardiac progenitor cell-derived exosomes prevent cardiomyocytes apoptosis through exosomal miR-21 by targeting PDCD4. Cell Death Dis. (2016) 7:e2277. doi: 10.1038/cddis.2016.181

61. Liu Y, Nie H, Zhang K, Ma D, Yang G, Zheng Z, et al. A feedback regulatory loop between HIF-1alpha and miR-21 in response to hypoxia in cardiomyocytes. FEBS Lett. (2014) 588:3137-46. doi: 10.1016/j.febslet.2014.05.067

62. Zlabinger K, Spannbauer A, Traxler D, Gugerell A, Lukovic D, Winkler J, et al. MiR-21, MiR-29a, GATA4, and MEF2c expression changes in endothelin-1 and angiotensin II cardiac hypertrophy stimulated Isl$1(+)$ Sca-1(+)c-kit(+) porcine cardiac progenitor cells in vitro. Cells. (2019) 8:1416. doi: $10.3390 /$ cells8111416

63. Yang C, Li B, Liu Y, Xing Y. Ginsenoside Rb1 protects cardiomyocytes from oxygen-glucose deprivation injuries by targeting microRNA-21. Exp Ther Med. (2019) 17:3709-16. doi: 10.3892/etm.2019.7330

64. Zhou X, Chang B, Gu Y. MicroRNA-21 abrogates palmitateinduced cardiomyocyte apoptosis through caspase-3/NFkappaB signal pathways. Anatol J Cardiol. (2018) 20:33646. doi: 10.14744/AnatolJCardiol.2018.03604

65. Gao Y, Huang R, Chen R, Li J, Luo W. Ischemic postconditioning altered microRNAs in human valve replacement. J Surg Res. (2016) 200:2835. doi: 10.1016/j.jss.2015.07.010

66. Yamada H, Suzuki K, Fujii R, Kawado M, Hashimoto S, Watanabe Y, et al. Circulating miR-21, miR-29a, and miR-126 are associated with premature death risk due to cancer and cardiovascular disease: the JACC Study. Sci Rep. (2021) 11:5298. doi: 10.1038/s41598-021-84707-7

67. Hanninen $M$, Jantti $T$, Tolppanen $H$, Segersvard $H$, Tarvasmaki $T$, Lassus J, et al. Association of miR-21-5p, miR-122-5p, and miR-320a$3 \mathrm{p}$ with 90-day mortality in cardiogenic shock. Int J Mol Sci. (2020) 21:7925. doi: 10.3390/ijms21217925

68. Bejerano T, Etzion S, Elyagon S, Etzion Y, Cohen S. Nanoparticle delivery of miRNA-21 mimic to cardiac macrophages improves myocardial remodeling after myocardial infarction. Nano Lett. (2018) 18:588591. doi: 10.1021/acs.nanolett.8b02578

69. Qiao L, Hu S, Liu S, Zhang H, Ma H, Huang K, et al. microRNA21-5p dysregulation in exosomes derived from heart failure patients impairs regenerative potential. J Clin Invest. (2019) 129:2237-50. doi: 10.1172/JCI123135

70. Cheng Y, Zhu P, Yang J, Liu X, Dong S, Wang $X$, et al. Ischaemic preconditioning-regulated miR-21 protects heart against ischaemia/reperfusion injury via anti-apoptosis through its target PDCD4. Cardiovasc Res. (2010) 87:431-9. doi: 10.1093/cvr/cvq082

71. Liu YL, Wu W, Xue Y, Gao M, Yan Y, Kong Q, et al. MicroRnA21 and-146b are involved in the pathogenesis of murine viral myocarditis by regulating TH-17 differentiation. Arch Virol. (2013) 158:1953-63. doi: 10.1007/s00705-013-1695-6

72. Shi B, Wang Y, Zhao R, Long X, Deng W, Wang Z. Bone marrow mesenchymal stem cell-derived exosomal miR-21 protects C-kit+ cardiac stem cells from oxidative injury through the PTEN/PI3K/Akt axis. PLoS ONE. (2018) 13:e0191616. doi: 10.1371/journal.pone.0191616

73. Li D, Mao C, Zhou E, You J, Gao E, Han Z, et al. MicroRNA-21 mediates a positive feedback on angiotensin II-induced myofibroblast transformation. $J$ Inflamm Res. (2020) 13:1007-20. doi: 10.2147/JIR.S285714

74. Thum T, Gross C, Fiedler J, Fischer T, Kissler S, Bussen M, et al. MicroRNA21 contributes to myocardial disease by stimulating MAP kinase signalling in fibroblasts. Nature. (2008) 456:980-4. doi: 10.1038/nature07511

75. Watanabe K, Narumi T, Watanabe $T$, Otaki Y, Takahashi T, Aono $\mathrm{T}$, et al. The association between microRNA-21 and hypertension-induced cardiac remodeling. PLoS ONE. (2020) 15:e0226053. doi: 10.1371/journal.pone.0226053
76. Shi B, Deng W, Long X, Zhao R, Wang Y, Chen W, et al. miR-21 increases c-kit $(+)$ cardiac stem cell proliferation in vitro through PTEN/PI3K/Akt signaling. PeerJ. (2017) 5:e2859. doi: 10.7717/peerj.2859

77. He F, Xiao Z, Yao H, Li S, Feng M, Wang W, et al. The protective role of microRNA-21 against coxsackievirus B3 infection through targeting the MAP2K3/P38 MAPK signaling pathway. J Transl Med. (2019) 17:335. doi: 10.1186/s12967-019-2077-y

78. Disertori M, Mase M, Ravelli F. Myocardial fibrosis predicts ventricular tachyarrhythmias. Trends Cardiovasc Med. (2017) 27:363-72. doi: 10.1016/j.tcm.2017.01.011

79. Jellis C, Martin J, Narula J, Marwick TH. Assessment of nonischemic myocardial fibrosis. J Am Coll Cardiol. (2010) 56:89-97. doi: 10.1016/j.jacc.2010.02.047

80. Graham-Brown MP, Patel AS, Stensel DJ, March DS, Marsh AM, McAdam J, et al. Imaging of myocardial fibrosis in patients with end-stage renal disease: current limitations and future possibilities. Biomed Res Int. (2017) 2017:5453606. doi: 10.1155/2017/5453606

81. Vivar R, Humeres C, Munoz C, Boza P, Bolivar S, Tapia F, et al. FoxO1 mediates TGF-beta1-dependent cardiac myofibroblast differentiation. Biochim Biophys Acta. (2016) 1863:128-38. doi: 10.1016/j.bbamcr.2015.10.019

82. Nonaka CKV, Sampaio GL, Silva KN, Khouri R, Macedo CT, Chagas Translational Research Consortium, et al. Therapeutic miR-21 silencing reduces cardiac fibrosis and modulates inflammatory response in chronic chagas disease. Int J Mol Sci. (2021) 22:3307. doi: 10.3390/ijms22073307

83. Hayashi H, Abdollah S, Qiu Y, Cai J, Xu YY, Grinnell BW, et al. The MAD-related protein Smad7 associates with the TGFbeta receptor and functions as an antagonist of TGFbeta signaling. Cell. (1997) 89:116573. doi: $10.1016 /$ S0092-8674(00)80303-7

84. Huebert RC, Li Q, Adhikari N, Charles NJ, Han X, Ezzat MK, et al. Identification and regulation of Sproutyl, a negative inhibitor of the ERK cascade, in the human heart. Physiol Genomics. (2004)18:2849. doi: 10.1152/physiolgenomics.00098.2004

85. Qiao G, Xia D, Cheng Z, Zhang G. Role of Sprouty1 (Spry1) in the pathogenesis of atrial fibrosis. Pathol Res Pract. (2018) 214:30813. doi: $10.1016 /$ j.prp.2017.04.021

86. Olivieri F, Spazzafumo L, Santini G, Lazzarini R, Albertini MC, Rippo MR, et al. Age-related differences in the expression of circulating microRNAs: miR-21 as a new circulating marker of inflammaging. Mech Ageing Dev. (2012) 133:675-85. doi: 10.1016/j.mad.2012.09.004

87. Bei Y, Wu X, Cretoiu D, Shi J, Zhou Q, Lin S, et al. miR-21 suppression prevents cardiac alterations induced by d-galactose and doxorubicin. J Mol Cell Cardiol. (2018) 115:130-41. doi: 10.1016/j.yjmcc.2018.01.007

88. Wang H, Bei Y, Shen S, Huang P, Shi J, Zhang J, et al. miR-21-3p controls sepsis-associated cardiac dysfunction via regulating SORBS2. J Mol Cell Cardiol. (2016) 94:43-53. doi: 10.1016/j.yjmcc.2016.03.014

89. Biomarkers Definitions Working Group. Biomarkers and surrogate endpoints: preferred definitions and conceptual framework. Clin Pharmacol Ther. (2001) 69:89-95. doi: 10.1067/mcp.2001.113989

90. Wang J, Tan GJ, Han LN, Bai YY, He M, Liu HB. Novel biomarkers for cardiovascular risk prediction. J Geriatr Cardiol. (2017) 14:13550. doi: 10.11909/j.issn.1671-5411.2017.02.008

91. Aronson JK, Ferner RE. Biomarkers-a general review. Curr Protoc Pharmacol. (2017) 76:9.23.1-17. doi: 10.1002/cpph.19

92. Landgraf P, Rusu M, Sheridan R, Sewer A, Iovino N, Aravin A, et al. A mammalian microRNA expression atlas based on small RNA library sequencing. Cell. (2007) 129:1401-14. doi: 10.1016/j.cell.2007.04.040

93. Sheedy FJ. Turning 21: induction of miR-21 as a key switch in the inflammatory response. Front Immunol. (2015) 6:19. doi: $10.3389 /$ fimmu.2015.00019

94. Jenike AE, Halushka MK. miR-21: a non-specific biomarker of all maladies. Biomark Res. (2021) 9:18. doi: 10.1186/s40364-021-00272-1

95. Lindow M, Kauppinen S. Discovering the first microRNA-targeted drug. $J$ Cell Biol. (2012) 199:407-12. doi: 10.1083/jcb.201208082

96. Bonneau E, Neveu B, Kostantin E, Tsongalis GJ, De Guire V. How close are miRNAs from clinical practice? A perspective on the diagnostic and therapeutic market. EJIFCC. (2019) 30:114-27. 
97. Huang CK, Bar C, Thum T. miR-21, Mediator, and potential therapeutic target in the cardiorenal syndrome. Front Pharmacol. (2020) 11:726. doi: 10.3389/fphar.2020.00726

98. Fujita S, Ito T, Mizutani T, Minoguchi S, Yamamichi N, Sakurai K, et al. miR-21 Gene expression triggered by AP-1 is sustained through a double-negative feedback mechanism. J Mol Biol. (2008) 378:492504. doi: 10.1016/j.jmb.2008.03.015

99. Loffler D, Brocke-Heidrich K, Pfeifer G, Stocsits C, Hackermuller J, Kretzschmar AK, et al. Interleukin-6 dependent survival of multiple myeloma cells involves the Stat3-mediated induction of microRNA21 through a highly conserved enhancer. Blood. (2007) 110:13303. doi: 10.1182/blood-2007-03-081133

100. Talotta F, Cimmino A, Matarazzo MR, Casalino L, De Vita G, D'Esposito $\mathrm{M}$, et al. An autoregulatory loop mediated by miR-21 and PDCD4 controls the AP-1 activity in RAS transformation. Oncogene. (2009) 28:7384. doi: 10.1038 /onc. 2008.370

101. Huang GL, Zhang XH, Guo GL, Huang KT, Yang KY, Hu XQ. Expression of microRNA-21 in invasive ductal carcinoma of the breast and its association with phosphatase and tensin homolog deleted from chromosome expression and clinicopathologic features. Zhonghua Yi Xue Za Zhi. (2008) 88:28337. doi: 10.3321/j.issn:0376-2491.2008.40.008

102. Seike M, Goto A, Okano T, Bowman ED, Schetter AJ, Horikawa I, et al. MiR-21 is an EGFR-regulated anti-apoptotic factor in lung cancer in never-smokers. Proc Natl Acad Sci USA. (2009) 106:1208590. doi: 10.1073/pnas.0905234106

103. Velu CS, Baktula AM, Grimes HL. Gfil regulates miR-21 and miR-196b to control myelopoiesis. Blood. (2009) 113:47208. doi: 10.1182/blood-2008-11-190215

104. Wickramasinghe NS, Manavalan TT, Dougherty SM, Riggs KA, Li Y, Klinge CM. Estradiol downregulates miR-21 expression and increases miR-21 target gene expression in MCF-7 breast cancer cells. Nucleic Acids Res. (2009) 37:2584-95. doi: 10.1093/nar/gkp117

105. Davis BN, Hilyard AC, Lagna G, Hata A. SMAD proteins control DROSHA-mediated microRNA maturation. Nature. (2008) 454:56-61. doi: 10.1038/nature07086

106. Du J, Yang S, An D, Hu F, Yuan W, Zhai C, et al. BMP-6 inhibits microRNA21 expression in breast cancer through repressing deltaEF1 and AP-1. Cell Res. (2009) 19:487-96. doi: 10.1038/cr.2009.34

107. Hao L, Wang J, Liu N. Long noncoding RNA TALNEC2 regulates myocardial ischemic injury in $\mathrm{H} 9 \mathrm{c} 2$ cells by regulating miR-21/PDCD4medited activation of Wnt/beta-catenin pathway. J Cell Biochem. (2019) 120:12912-23. doi: 10.1002/jcb.28562

108. Wu Z, He Y, Li D, Fang $\mathrm{X}$, Shang $\mathrm{T}$, Zhang $\mathrm{H}$, et al. Long noncoding RNA MEG3 suppressed endothelial cell proliferation and migration through regulating miR-21. Am J Transl Res. (2017) 9:3326-35.

109. Nag AC. Study of non-muscle cells of the adult mammalian heart: a fine structural analysis and distribution. Cytobios. (1980) 28:41-61.

110. Zhou P, Pu WT. Recounting cardiac cellular composition. Circ Res. (2016) 118:368-70. doi: 10.1161/CIRCRESAHA.116.308139

111. Bronze-da-Rocha E. MicroRNAs expression profiles in cardiovascular diseases. Biomed Res Int. (2014) 2014:985408. doi: 10.1155/2014/985408

112. Bonci D. MicroRNA-21 as therapeutic target in cancer and cardiovascular disease. Recent Pat Cardiovasc Drug Discov. (2010) 5:156-61. doi: 10.2174/157489010793351962

113. Zhou SS, Jin JP, Wang JQ, Zhang ZG, Freedman JH, Zheng Y, et al. miRNAS in cardiovascular diseases: potential biomarkers, therapeutic targets and challenges. Acta Pharmacol Sin. (2018) 39:1073-84. doi: 10.1038/aps.2018.30

114. Chistiakov DA, Orekhov AN, Bobryshev YV. Cardiacspecific miRNA in cardiogenesis, heart function, and cardiac pathology (with focus on myocardial infarction). $J \mathrm{Mol}$ Cell Cardiol. (2016) 94:107-21. doi: 10.1016/j.yjmcc.2016. 03.015

115. Huang Y, Li J. MicroRNA208 family in cardiovascular diseases: therapeutic implication and potential biomarker. J Physiol Biochem. (2015) 71:47986. doi: 10.1007/s13105-015-0409-9

116. Ajuyah P, Hill M, Ahadi A, Lu J, Hutvagner G, Tran N. MicroRNA (miRNA)to-miRNA regulation of programmed cell death 4 (PDCD4). Mol Cell Biol. (2019) 39:e00086-19. doi: 10.1128/MCB.00086-19

Conflict of Interest: The authors declare that the research was conducted in the absence of any commercial or financial relationships that could be construed as a potential conflict of interest.

Publisher's Note: All claims expressed in this article are solely those of the authors and do not necessarily represent those of their affiliated organizations, or those of the publisher, the editors and the reviewers. Any product that may be evaluated in this article, or claim that may be made by its manufacturer, is not guaranteed or endorsed by the publisher.

Copyright (c) 2021 Surina, Fontanella, Scisciola, Marfella, Paolisso and Barbieri. This is an open-access article distributed under the terms of the Creative Commons Attribution License (CC BY). The use, distribution or reproduction in other forums is permitted, provided the original author(s) and the copyright owner(s) are credited and that the original publication in this journal is cited, in accordance with accepted academic practice. No use, distribution or reproduction is permitted which does not comply with these terms. 